\title{
Daily breathing inconsistency in pancreas SBRT: a 4DCT study
}

\author{
Vikren Sarkar, Shane Lloyd, Adam Paxton, Long Huang, Fan-Chi Su, Randa Tao, Jonathan Tward, Hui \\ Zhao, Bill Salter
}

Department of Radiation Oncology, University of Utah Huntsman Cancer Hospital, Salt Lake City, UT, USA

Contributions: (I) Conception and design: V Sarkar, S Lloyd, B Salter, J Tward, R Tao; (II) Administrative support: All authors; (III) Provision of study materials or patients: S Lloyd, R Tao; (IV) Collection and assembly of data: V Sarkar, S Lloyd, B Salter; (V) Data analysis and interpretation: All authors; (VI) Manuscript writing: All authors; (VII) Final approval of manuscript: All authors.

Correspondence to: Vikren Sarkar. Division of Medical Physics, Department of Radiation Oncology, University of Utah Huntsman Cancer Hospital, 1950 Circle of Hope, Salt Lake City, UT 84112, USA. Email: vikren.sarkar@hci.utah.edu.

\begin{abstract}
Background: Stereotactic body radiation therapy (SBRT) treatments of pancreatic cancer typically employ relatively small margins. This study characterizes the motion of high visibility structures in close proximity to the pancreas to determine how much the motion envelope of such a structure changes due to respiratory variation between fractions.

Methods: Fanbeam, four-dimensional computed tomography (4DCT) studies acquired initially for planning and again prior to each treatment for 6 patients were used to fully characterize the change in motion of high-contrast structures in close proximity to the pancreas.

Results: Three of the six patients investigated had structures that showed a change in motion over the course of treatment that would not have been covered when using the typical $3 \mathrm{~mm}$ planning target volume (PTV) margins. For most of these large changes in motion envelope, a $4 \mathrm{~mm}$ uniform PTV margin would have allowed for coverage of the tumor.

Conclusions: Half of the patients showed a change in motion envelope greater than would be covered by the commonly used PTV margins in pancreas SBRT. This shows that the impact of small margins must be very carefully considered during the planning process.
\end{abstract}

Keywords: Pancreas stereotactic body radiation therapy (pancreas SBRT); planning target volume margins (PTV margins); respiratory motion

Submitted May 07, 2018. Accepted for publication Sep 17, 2018.

doi: 10.21037/jgo.2018.09.08

View this article at: http://dx.doi.org/10.21037/jgo.2018.09.08

\section{Introduction}

According to the latest estimates (1), it is expected that pancreatic cancer will soon represent one of the most commonly diagnosed gastrointestinal (GI) cancers after colorectal cancer, and will represent the fourth leading cause of cancer-related deaths. For those patients diagnosed with locally-advanced pancreatic cancer (LAPC), the typical treatment approach consists of chemotherapy followed by radiation therapy in appropriately selected patients (2). The median survival for such treatment approaches is $9-13$ months (2), and 5-year overall survival (OS) is typically only $3 \%(1)$. Thus, there is significant interest in finding ways to improve treatment options for these patients. One approach includes investigating ways to utilize advances in technology to improve the radiation treatment and delivery. It is well-known that pancreas adenocarcinomas are radioresistant, with low rates of pathologic response to conventional chemoradiation therapy $(3,4)$. It was recently reported that the use of advanced imaging and radiation planning allowed for dose escalation beyond conventional chemoradiation therapy, which was found to be associated with improvements in both OS and local-regional recurrence-free survival (2). Unfortunately, the surrounding normal structures have dose limiting toxicities that make it difficult to deliver the higher radiation doses that seem to 
be associated with improved disease control in pancreatic cancer. Techniques such as stereotactic body radiation therapy (SBRT) and intensity modulated 'dose painting' are being actively investigated to overcome these challenges $(5,6)$.

When designing a radiotherapy plan for targets located in the upper GI area, it is recognized that those structures (both target and organs at risk) in close proximity to the diaphragm experience motion due to respiration. One common approach to treat such patients is to use 4DCT to fully characterize the motion of the target through generation of an internal target volume (ITV), which is then further expanded into a planning target volume (PTV) to account for such factors as setup inaccuracies. Given the location of the target for LAPC, and its proximity to a large number of radiosensitive organs, a highly conformal dose distribution must be used in conjunction with small PTV margins (5).

While it is well known that patients' breathing can change from day to day $(7,8)$, the effect of this change on the ITV has not been fully characterized, especially in the context of the pancreas. Given the small margins used for SBRT of the pancreas, it is unclear whether potential changes in motion envelope due to variation in breathing can be fully accounted for by the original, simulationdetermined ITV, and the PTV margins alone. From 2015 to 2017, our clinical workflow for all pancreas SBRT patients included obtaining fanbeam 4-dimensional computed tomography (4DCT) scans for image guidance before every delivered fraction. In this analysis we analyzed these 4DCTs to characterize the change in motion envelope of representative structures in close proximity to the pancreas tumors. Our goal was to assess whether the observed interfractional change in breathing motion could result in marginal misses of radiation delivery when using a uniform PTV expansion of $3 \mathrm{~mm}$.

\section{Methods}

In a previously utilized clinical workflow for SBRT in our department, a fanbeam 4DCT was acquired for image guidance purposes prior to the delivery of each treatment fraction. For this project, we identified all patients treated by SBRT for pancreatic cancer that used that workflow. All 6 of these patients were treated after initial chemotherapy. No patients underwent surgical resection prior to treatment but some had surgical clips from biopsies or other procedures. For five patients, the prescription dose was 33 Gy in 5 fractions. For one patient (case 4), the prescription was $25 \mathrm{~Gy}$ in 5 fractions. At the time of treatment for these patients, the workflow did not include placement of fiducial markers.

All patients were immobilized using a BodyFIX fullbody, double vacuum bag (Elekta AB, Stockholm, Sweden). All 4DCT scans (one for planning purposes and one immediately prior to each fraction) were obtained using cine mode on a Lightspeed16RT scanner (GE Healthcare, Waukesha, WI, USA). The RPM device (version 1.6.5, Varian Medical Systems, Palo Alto, CA, USA) was used to track the patient's breathing, and the images were binned into 10 separate phases. All CTs were obtained using $2.5 \mathrm{~mm}$ slice thickness, $120 \mathrm{kVp}$ and the auto-mA setting. The FOV was manually set using the scout images (localizer scans) to cover the full extent of the bag and patient. Since this was a manual process, the FOV ranged from 59 to $62 \mathrm{~cm}$, resulting in axial pixel dimensions of 1.21-1.15 mm. No further breathing control technique was used during CT acquisition.

For all 6 patients investigated here, the 4DCT datasets acquired were transferred to the MIM software (MIM Maestro, version 6.6.8, MIM Software Inc, Cleveland, $\mathrm{OH}, \mathrm{USA})$. This represented a total of 35 datasets used for this analysis - one of the patients did not have a 4DCT on his last fraction due to equipment malfunction. Each of the 4DCT datasets was then used to generate a maximum intensity projection (MIP) image set that was also used for this analysis.

For each patient, an easily identifiable structure [stent (3 patients), surgical clip ( 2 patients), calcification (1 patient)] located within $1 \mathrm{~cm}$ of the target volume was identified on the MIP dataset created from the simulation 4DCT. These high visibility objects were used, instead of the nearby tumor, in order to eliminate the introduction of positional misrepresentation error that might occur if the tumor were used. The window-level was set to the "soft-tissue" preset within MIM (level =40, window =400). The movement of the highly visible object in the anterior-posterior (AP) and left-right (LR) directions was characterized by scrolling through all the axial CT slices and determining the DICOM coordinates of object points with the maximum extent of motion in the direction of interest. This was repeated using the sagittal images to determine the extent of motion in the superior/inferior (SI) direction. For each subsequent dataset for that patient, the same object was identified and the previously-described steps were repeated to characterize the motion envelope 
Table 1 Summary of extent of motion of solid objects located within close proximity to the PTV over six imaging studies

\begin{tabular}{|c|c|c|c|c|c|c|c|c|c|c|}
\hline \multirow{2}{*}{ Patient } & \multirow{2}{*}{ Object } & \multicolumn{3}{|c|}{ Minimum extent of motion $(\mathrm{mm})$} & \multicolumn{3}{|c|}{ Maximum extent of motion $(\mathrm{mm})$} & \multicolumn{3}{|c|}{ Maximum change in extent of motion $(\mathrm{mm})$} \\
\hline & & LR & AP & SI & LR & AP & SI & LR & AP & SI \\
\hline 1 & Stent & 28.4 & 30.7 & 47.4 & 35.4 & 34.9 & 53.8 & $7.0^{\star}$ & 4.2 & $6.4^{*}$ \\
\hline 2 & Stent & 31.1 & 42.3 & 60.3 & 38.9 & 49.2 & 63.9 & $7.8^{\star}$ & $6.9^{\star}$ & 3.6 \\
\hline 3 & Stent & 34.4 & 23.5 & 67.8 & 38.6 & 26.2 & 84.8 & 4.2 & 2.7 & $17.0^{*}$ \\
\hline 4 & Surgical clip & 4.7 & 6.0 & 9.0 & 6.1 & 7.7 & 11.0 & 1.4 & 1.7 & 2.0 \\
\hline 5 & Calcification & 7.9 & 5.7 & 8.1 & 8.5 & 6.6 & 10.0 & 0.6 & 0.9 & 1.9 \\
\hline 6 & Surgical clip & 4.8 & 7.0 & 16.0 & 7.7 & 9.1 & 18.9 & 2.9 & 2.1 & 2.9 \\
\hline
\end{tabular}

*, motion range changes greater than $6.0 \mathrm{~mm}$. LR, left-right; AP, anterior-posterior; SI, superior/inferior.

of the high visibility object. The same window-level preset was used in all cases. Any change in the motion envelope of more than $6 \mathrm{~mm}$ would risk the new ITV not being fully covered by the $3 \mathrm{~mm}$ symmetric PTV margin that was used for patient treatment.

To investigate how often the change in motion envelope would lead to a partial miss of the target, two scenarios were investigated. First, each 4DCT acquired prior to the treatments were compared to the simulation 4DCT to determine how many times the motion envelope changed significantly enough $(6 \mathrm{~mm})$ to cause a partial miss of the target over the course of treatment. For this patient cohort, this represents a total of 29 cases to be investigated. Secondly, given that breathing changes occur randomly, an extension to this analysis is to consider any of the 4DCTs as being equally likely to have occurred during the planning process. This extended analysis leads to 175 possible scenarios to be evaluated.

In an effort to understand the relationship between tissue motion and respiratory trace, the breathing traces were further examined. It was postulated that fractions with atypical breathing traces would correlate with differences in the motion envelope of the object of interest.

\section{Results}

Table 1 summarizes the largest changes in motion envelope for each object studied here. The extent of motion reported represents the size of a motion envelope that would encompass the entire motion of the particular object. Therefore, larger objects, such as the stents, show larger motion envelopes.

On average, the maximum change in motion extent across all 6 4DCT data sets for each patient was 3.1, 3.0 and $4.5 \mathrm{~mm}$ in the LR, AP and SI directions respectively. The corresponding standard deviations were 2.7, 2.0 and $4.9 \mathrm{~mm}$. Patient 3 showed an extreme change of $17 \mathrm{~mm}$ in the SI direction while patient 5 had the smallest maximum change in S/I direction of $1.9 \mathrm{~mm}$.

Figure 1 shows the breathing traces obtained during the 4DCT acquisitions for each patient. The displayed traces show the RPM data from the start to end of actual CT data acquisition. For ease of comparison, the traces were renormalized so that they extend from -1 to 1 . The red areas within each curve represent the parts of the breathing cycle where the object under consideration was being imaged.

Half of the patients investigated in this study had objects whose maximum change in motion envelope exceeded the theoretical $6 \mathrm{~mm}$ threshold in motion change that could be accommodated by a PTV margin of $3 \mathrm{~mm}$. This includes one case where the maximum motion change was an extreme $17 \mathrm{~mm}$ in the SI direction (case 3). The breathing traces for case 3 from Figure 1 show several large spikes in breathing for some fractions. For this particular patient, the most motion happened during simulation, followed by treatment day 4 . The least motion happened during treatment day 2 followed by day 3. The breathing traces from figure 1 seem to be consistent with these observations in that the traces are quite irregular and variable, as might be expected to occur in coincidence with the variable structure motion that was observed. The average motion range from the RPM trace were smallest for treatment days 2 and 3 while it was highest for the simulation and treatment day 4.

Two of the 29 comparisons (6.9\%) done for the patients using the 4DCTs in the order they were acquired clinically showed changes in motion envelope greater than $6 \mathrm{~mm}$. Both occurred on the same patient (patient 2) and were in 


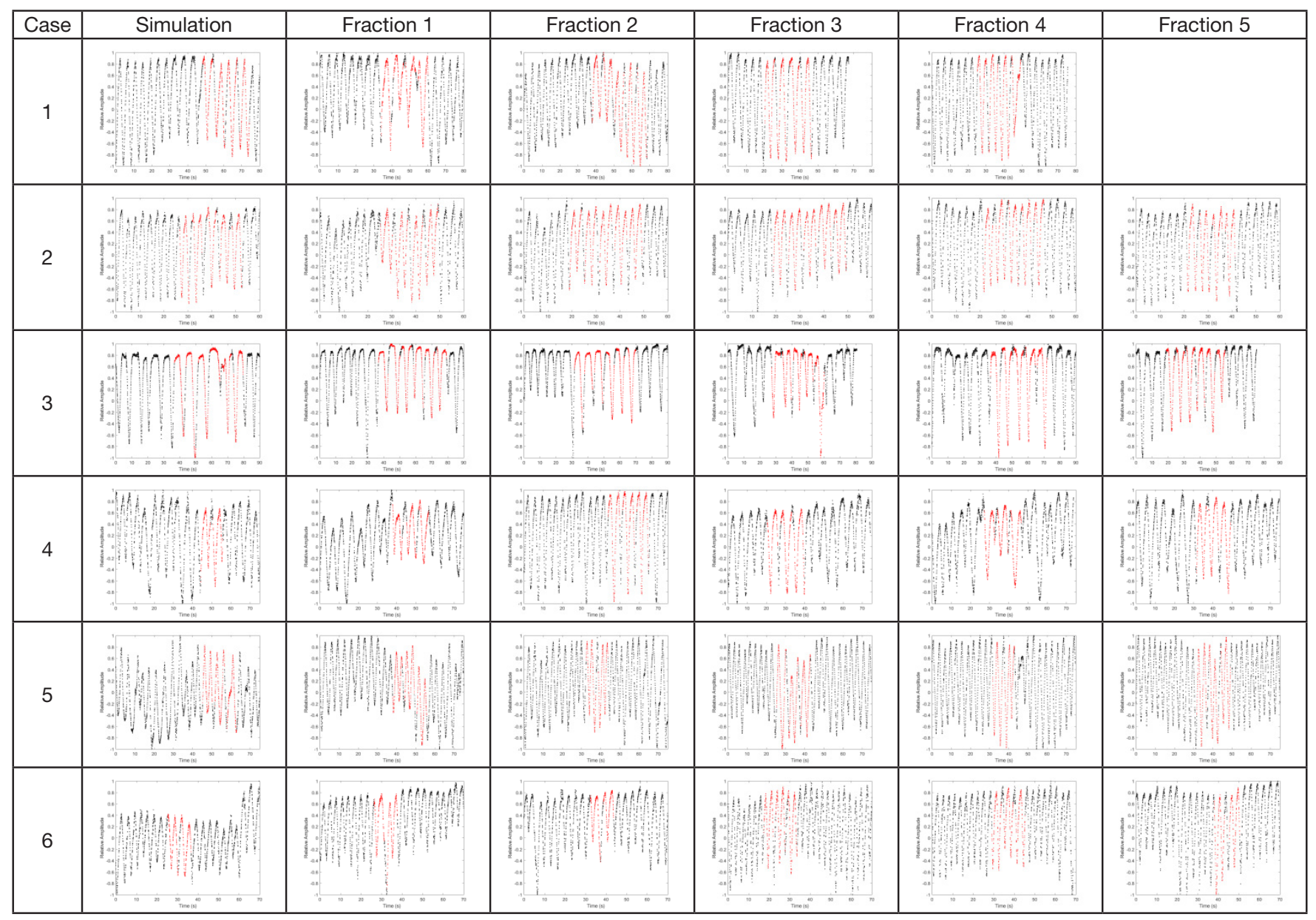

Figure 1 RPM traces for each dataset investigated. The red areas represent the trace at the time the objects under investigation were being imaged.

the AP direction. For the second scenario, where any of the 4DCTs was considered to be a possible simulation scan, 12 of the 175 comparisons (6.9\%) lead to a change greater than $6 \mathrm{~mm}$. The majority (8) occurred in the SI direction. One patient had a $\geq 6 \mathrm{~mm}$ change in both the LR and SI directions.

\section{Discussion}

Several past studies have shown that the motion of the pancreas is non-trivial and hard to characterize. In 2009, Minn et al. (9) tried to correlate the motion of fiducials during the planning 4DCT and the same motion during treatment. Not only did they find large motions of implanted fiducials in the 4DCT used for planning, but they also could not find a correlation between that motion and the motion of the same fiducials when using CyberKnife's tracking during treatment. One may surmise that what they were actually seeing were changes in the ITV second to changes in the patient's breathing between the planning session and the treatment sessions. More recently, Bae et al. (10) found that simple bony alignment was not sufficient when treating lesions of the pancreas.

Knowing that pancreatic targets experience non-trivial motion second to respiration, and that small PTV margins are typically used for treatment of such targets, we have endeavored to understand how much this respiratory driven target motion may change over the typical 1-2 weeks course of SBRT treatment. Conventional wisdom would suggest that PTV margins are designed to mitigate such challenges, but given the $3 \mathrm{~mm}$ margins typically used for pancreatic treatment we are mindful that variations in 
ITV extent of greater than $6 \mathrm{~mm}$ will completely consume the leeway provided by the PTV, and lead to risk of target under-dosage. We are further mindful that the PTV is also expected to mitigate setup inaccuracies, along with any other errors that may enter into the process. If the entirety of the PTV margin is accounted for by ITV variations alone, then it seems inevitable that some degree of tumor under dosage will occur.

In this study we used gold standard, fanbeam 4DCT data to characterize daily variations in breathing motion with a high degree of accuracy. Our results revealed that ITV variations of magnitude sufficient to exhaust a $3 \mathrm{~mm}$ PTV do, indeed, occur (with frequency of approximately $7 \%$ ). We observed that these daily variations appear to be related to variations in patient breathing pattern, as evidenced by visible, and sometimes extreme, variations in the patients breathing trace for that same day.

When considering the 4DCTs in the order they were acquired clinically for each patient, we observed that 2 of the 29 studied fractions (6.9\%) would have experienced a partial geometric miss of the target. Both of these fractions would have realized full coverage if a $4 \mathrm{~mm}$ PTV margin had been used. When considering all 175 possible combinations of simulation and treatment 4DCT's order, 12 fractions (6.9\%) would have led to partial geometric misses. For these situations, the use of a $4 \mathrm{~mm}$ margin would have provided for full coverage of 166 of the cases (95\%).

It is important to realize that even changes in the motion envelope that are smaller than $6 \mathrm{~mm}$ could lead to geometric misses, given inevitable uncertainties in the image registration process. For instance, if only a $1 \mathrm{~mm}$ image registration error was made (on the order of 1 pixel), then a change in motion envelope of only $4 \mathrm{~mm}$ in total motion envelope would lead to a partial geometric miss. In the presence of $1 \mathrm{~mm}$ simulated registration error, for our cohort of 6 patients, 29 4DCT's, partial geometric misses would have occurred in 5 of 29 cases $(17.3 \%)$ when considering 4DCTs in the order they were actually acquired, and in 31 of 175 cases $(17.7 \%)$ when all possible combinations of 4DCT orders were considered.

It should be pointed out that a previous study by Goldstein et al. (11) showed that the motion of the target for pancreatic lesions was not well correlated to the motion of biliary stents on any particular day. It is not our intent that biliary stents should be used as fiducial markers for targeting pancreas lesions. The present study is assessing how the degree and pattern of motion due to breathing in this area of the body changes from day to day. If the stent's motion shows significant changes, then we hypothesize that the target's motion is also differing on a day to day basis.

Although beyond the scope of this analysis, challenges in accurately delineating tumor extent should also be taken into account when determining the appropriate margin expansions for pancreas radiation therapy. In illustration of this point, prior to the present analysis, an attending physician specializing in GI radiation oncology contoured the ITV on the 4DCT from each treatment day for each of the patients above. However, it became evident that soft tissue discrimination on these non-contrasted scans was poor, subsequently resulting in inconsistent ITV contours, even when performed by the same physician. Published studies of inter-physician contouring variability also illustrate this point (12), and MRI-based planning has been shown to result in smaller contours than CT-based planning (13).

While we cannot assume that the motion changes measured here for high visibility objects adjacent to the target are directly assignable to the target itself, it is not unreasonable to expect that comparable, impactful changes in the motion of the GTV are likely, as well. The key question at hand is, whether the PTV margin used is large enough to still provide adequate coverage of the target.

Experience gained during this study suggests that daily ITV magnitude variations, driven by patient respiration pattern differences, may argue for consideration of increased PTV margins to fully cover pancreatic tumors throughout the course of SBRT treatment. We recognize that when high dose radiation is not deliverable to the entire extent of tumor, due to surrounding organs at risk, it may still be advantageous to deliver high dose radiation to the central tumor and/or the tumor vessel interface in order to improve the chances of local control and/or a successful surgical resection (14). Therefore, a strategy of delivering lower dose radiation, with wider margins, while boosting certain essential areas may be optimal.

The current study has some limitations that are important to discuss. As mentioned, intravenous contrast was not used for daily 4DCT control scans, so tumor delineation was difficult and we were unable to measure changes in the ITV shape or size directly. We used nearby high visibility structures as proxies for the tumor even though it is unknown to what extent their motion mirrors the tumor motion. We note, however, that because the structures used were of high contrast and, thus, easily delineated, the degree of accuracy with which we characterized the motion envelopes of these surrogate 
structures was high. We also note that in cases with stents and surgical clips, the high visibility structures were embedded within the target tumor area. Additionally, the treatment techniques used in this analysis did not include placement of fiducial markers, use of breath hold, or respiratory gating, and the results of this analysis apply only when free breathing techniques are used. Another potential limitation to the study is that only 6 patients met the study inclusion criteria. As with any study with a small number of patients, it may be difficult to generalize our results to a wider setting. However, this study did include a large number of high quality, fanbeam 4DCT datasets, with a total of 35 separate scans that quantified the variation in motion for each fraction of radiation therapy. With these many datasets, across multiple patients, our findings can contribute to the accurate quantification of ITV variation in the treatment of pancreas cancer with SBRT and, as such, should serve to inform further discussions regarding the optimal magnitude of PTV margins used in such cases.

\section{Conclusions}

We observed significant, respiration induced variations in tissue motion magnitude in the vicinity of a radiation targeted pancreatic tumor. When free-breathing techniques were used, a $3 \mathrm{~mm}$ PTV margin was not sufficient to account for these daily ITV variations, leading to partial geometric misses for some treatment fractions. When using freebreathing techniques for the treatment of pancreatic cancer, PTV margins of greater than $3 \mathrm{~mm}$ should be considered if the intent is to deliver full prescribed dose to the entire tumor/target extent. In our cohort of patients, a $4 \mathrm{~mm}$ margin would have provided coverage for the observed ITV variation in $95 \%$ of the treated fractions. Because of the daily variation in respiration induced tumor motion observed here, careful consideration should be given to PTV margin selection.

\section{Acknowledgements}

None.

\section{Footnote}

Conflicts of Interest: The authors have no conflicts of interest to declare.

Ethical Statement: The study was covered under a University of Utah IRB-approved study allowing retrospective analysis of patient imaging data. All data was collected retrospectively, and the study was deemed to represent minimal risk to patients, so informed consent was not obtained; however, patients' data has been secured according to requirements of IRB protocol.

\section{References}

1. American Cancer Society. Cancer Facts \& Figures 2018: Atlanta: American Cancer Society 2018. Available online: https://www.cancer.org/research/cancer-facts-statistics/allcancer-facts-figures/cancer-facts-figures-2018.html

2. Krishnan S, Chadha AS, Suh Y, et al. Focal Radiation Therapy Dose Escalation Improves Overall Survival in Locally Advanced Pancreatic Cancer Patients Receiving Induction Chemotherapy and Consolidative Chemoradiation. Int J Radiat Oncol Biol Phys 2016;94:755-65.

3. Evans DB, Varadhachary GR, Crane $\mathrm{CH}$, et al. Preoperative gemcitabine-based chemoradiation for patients with resectable adenocarcinoma of the pancreatic head. J Clin Oncol 2008;26:3496-502.

4. Zhao Q, Rashid A, Gong Y, et al. Pathologic complete response to neoadjuvant therapy in patients with pancreatic ductal adenocarcinoma is associated with a better prognosis. Ann Diagn Pathol 2012;16:29-37.

5. Herman JM, Chang DT, Goodman KA, et al. Phase 2 multi-institutional trial evaluating gemcitabine and stereotactic body radiotherapy for patients with locally advanced unresectable pancreatic adenocarcinoma. Cancer 2015;121:1128-37.

6. Quan K, Sutera P, Xu K, et al. Results of a prospective phase 2 clinical trial of induction gemcitabine/capecitabine followed by stereotactic ablative radiation therapy in borderline resectable or locally advanced pancreatic adenocarcinoma. Pract Radiat Oncol 2018;8:95-106.

7. Hugo G, Vargas C, Liang J, et al. Changes in the respiratory pattern during radiotherapy for cancer in the lung. Radiother Oncol 2006;78:326-31.

8. Seppenwoolde Y, Shirato H, Kitamura K, et al. Precise and real-time measurement of $3 \mathrm{D}$ tumor motion in lung due to breathing and heartbeat, measured during radiotherapy. Int J Radiat Oncol Biol Phys 2002;53:822-34.

9. Minn AY, Schellenberg D, Maxim P, et al. Pancreatic tumor motion on a single planning 4D-CT does not correlate with intrafraction tumor motion during treatment. Am J Clin Oncol 2009;32:364-8. 
10. Bae JS, Kim DH, Kim WT, et al. The role of surgical clips in the evaluation of interfractional uncertainty for treatment of hepatobiliary and pancreatic cancer with postoperative radiotherapy. Radiat Oncol J 2017;35:65-70.

11. Goldstein SD, Ford EC, Duhon M, et al. Use of respiratory-correlated four-dimensional computed tomography to determine acceptable treatment margins for locally advanced pancreatic adenocarcinoma. Int J Radiat Oncol Biol Phys 2010;76:597-602.

12. Holyoake DL, Robinson M, Grose D, et al. Conformity analysis to demonstrate reproducibility of target volumes for Margin-Intense Stereotactic Radiotherapy for borderline-resectable pancreatic cancer. Radiother Oncol

Cite this article as: Sarkar V, Lloyd S, Paxton A, Huang L, Su FC, Tao R, Tward J, Zhao H, Salter B. Daily breathing inconsistency in pancreas SBRT: a 4DCT study. J Gastrointest Oncol 2018;9(6):989-995. doi: 10.21037/jgo.2018.09.08
2016;121:86-91.

13. Hall WA, Heerkens HD, Paulson ES, et al. Pancreatic gross tumor volume contouring on computed tomography (CT) compared with magnetic resonance imaging (MRI): Results of an international contouring conference. Pract Radiat Oncol 2018;8:107-15.

14. Combination Chemotherapy With or Without Hypofractionated Radiation Therapy Before Surgery in Treating Patients With Pancreatic Cancer. In: ClinicalTrials.gov. 2018. Available online: https://clinicaltrials.gov/ct2/show/ NCT02839343 ?term $=021501 \&$ rank $=1$ 